\title{
Occult cerebral arteriovenous malformations
}

\author{
P TSITSOPOULOS, J ANDREW, M J G HARRISON \\ From the Department of Neurological Studies, The Middlesex Hospital Medical School, London, UK
}

SUMMARY Angiographically occult arteriovenous malformations in the brain are rare but cause considerable diagnostic difficulty. Four personal cases are presented in which CT scans showed hypodense lesions with minimal or no enhancement, and angiograms showed only an avascular mass. Gliomas, a dermoid and an infarct were suspected before histological examination of biopsies or excised specimens. The patients had presented in early adult life with epilepsy and focal deficits.

The great majority of cerebral vascular malformations are arteriovenous malformations (AVMs) and are detected by angiography. ${ }^{1-3}$ However, in 1985 Hashim et $\mathrm{al}^{4}$ were able to find 47 cases in the literature in which angiography had failed to reveal such a lesion. They suggested that a hyperdense lesion on CT scanning was the usual finding and was the essential clue to the pre-operative diagnosis. This has not been our experience and so we are reporting four consecutive cases referred to one of us (JA) in recent years.

\section{Case reports}

Case 1 This 37 year old man had had a single grand mal seizure in his sleep in April 1979. Within 5 months and despite phenytoin he had developed an aura of nausea and abdominal discomfort before his attacks which now began with shaking of the right hand. After one of them he noted right sided limb weakness for several minutes. Examination revealed a little hesitancy of speech and an extensor plantar response on the right. There was a left anterior quadrant abnormality on the EEG, and an unenhanced CT scan revealed a low density area showing a mass effect above the anterior horn of the left lateral ventricle. Carotid angiography confirmed the mass which was avascular. A glioma was suspected. At surgery a soft area was encountered which was excised and proved to contain abnormal dilated vessels of various sizes with thick collagenous walls lined by a single layer of endothelial cells. The vessels contained no muscle cell layer.They were closely packed with no glial elements interposed. There were areas of gliosis and oedema in the

Address for reprint requests: Dr MJG Harrison, Department of Neurological Studies, The Middlesex Hospital Medical School, Mortimer Street, London, W1N 8AA, UK.

Received 3 April 1986 and in revised form 16 June 1986. Accepted 23 June 1986 white matter and some haemosiderin deposits. There was also evidence of recent haemorrhage. A cavernous angioma was diagnosed. Post-operatively seizure control was readily obtained, and he had no further fits over the next 5 years of follow up.

Case 2 A 38 year old man had been under the care of the Department of Psychiatry with a personality disorder for 9 or 10 years. This had in recent years been complicated by heavy drinking. In March 1979 he felt unwell with a flu-like illness and did not drink any alcohol for $\mathbf{4 8}$ hours. He then had two seizures and was admitted in a restless, tremulous, disorientated state. He became paranoid and was diagnosed as suffering from an alcohol withdrawal state. He continued to have further seizures over the next 2 weeks. Examination revealed a mild dysphasia with absent right-sided abdominal reflexes. An EEG, however, showed right frontal seizure activity. A CT scan showed a bilateral medial frontal low density lesion with only minimal enhancement. Angiography showed the lesion to be avascular. A frontal glioma was diagnosed. At operation the right frontal area was needled. A soft area was detected and included in a standard right frontal lobectomy. The specimen contained oedematous and gliotic white matter containing small foci of calcification, and enlarged abnormal arteries and veins. The vascular channels had thick walls with fibrous thickening of the intima, areas of absent elastic lamina, thickening of the muscle layer, and fibrosis of the adventitia. Some of the smaller vessels were thrombosed. The arteriovenous malformation was surrounded by fibrosis and by macrophages containing haemosiderin, but there was no evidence of recent haemorrhage. He had only one post-operative seizure but had some residual naming difficulty.

Case 3 This 36 year old man had had epilepsy since the age of 1 year. At the age of 12, the nature of his attacks changed and he began to experience episodes of uncontrollable fear and crying lasting for up to half an hour. By the time he was 21 , attacks were preceded by paraesthesiae beginning in the left hand, spreading up the arm and affecting the left side of the mouth, he then heard a snatch of unfamiliar music, the whole episode lasting 5 minutes. Phenytoin failed to control his epilepsy. In 1975 (aged 32) he developed some left-sided 
weakness and a right parietal cyst was tapped at a craniotomy performed elsewhere. This was repeated the next year. When referred to the Middlesex Hospital he also complained of a hallucinatory blue object in the left visual field. Examination showed a slight left-sided hemiparesis and a right parietal craniotomy scar. An EEG showed slow wave activity over the site of the old craniotomy scar but no seizure changes. The CT scan showed a large rounded low density lesion in the right posterior temporal and parietal area which was slightly compressing the lateral ventricle. There was a small spot of calcification at its medial margin. The lesion did not enhance. Angiography showed an avascular parietal mass. The radiological diagnosis was of a developmental tumour such as an intracerebral dermoid. At surgery the mass proved to consist of a multilocular cyst which was drained and its wall was biopsied. Histological examination showed some thick walled abnormal blood vessels in a fibrous gliotic grey and white matter with some microscopic calcification. The blood vessels had no muscle layer and contained much collagen. They were very closely opposed and there was no glial tissue between them. An AVM was diagnosed.

Case 4 A 23 year old man became drowsy, complained of severe headache, and collapsed with a left hemiplegia. He was investigated elsewhere, where a lumbar puncture revealed no evidence of a subarachnoid haemorrhage. A craniotomy was carried out but no diagnosis was made. His general condition improved and 4 months later he had only a mild left-sided hemiparesis and hypaesthesia. An EEG showed diffuse right sided slow activity with occasional spikes. Plain skull radiographs showed no pressure changes. A CT scan showed a low density area in the right middle cerebral artery territory which was interpreted as a possible infarct. The right lateral ventricle was slightly dilated. Bilateral carotid angiography showed a paucity of branches of the right pericallosal and middle cerebral arteries. There was no neoplastic circulation, and no displacement of major vessels. Whilst carrying out an acrylic cranioplasty the opportunity was taken to perform a cortical biopsy. This revealed fragments of grey matter showing abnormal arteries and veins with hyperplastic walls. There was evidence of gliosis and microcalcifications between the vessels, some of which were thrombosed. The appearances were those of an arteriovenous malformation.

\section{Discussion}

Four young adults presented with epilepsy or a focal deficit and proved to have angiographically occult arteriovenous malformations. Review of the literature shows epilepsy to be the commonest manifestation. ${ }^{45}$ The history of seizures was of very varied duration in our small group of cases, however, and in one case these were initially attributed to alcohol withdrawal diverting attention away from the possibility of a structural lesion. In two others, the history revealed the appearance and evolution of focal features to their attacks leading to full neuroradiological examination. In the fourth case a sudden hemiplegia prompted investigation.

Skull radiographs are usually unreliable. Dilated vascular channels may be seen in large AVMs but no such findings are to be expected with these angiographically occult small malformations. EEGs are nearly always abnormal since many of these lesions are superficial and epileptogenic. The changes are however non-specific though focal seizure activity or slow wave disturbance does of course obligate one to arrange neuroradiological examination.

Previous accounts have stressed that the CT scan is very suggestive of the correct diagnosis since the lesion is slightly hyperdense, and in over $90 \%$ of cases shows enhancement. ${ }^{6}$ This has not been our experience. All four of our patients showed a low density area with some small mass effect mimicking a low grade astrocytoma, infarct or nonvascular mass lesion. Minimal enhancement was only detected in one of the three given a contrast agent. By definition angiography showed an avascular mass of unknown pathology.

The decision to operate on these patients was determined partly by their clinical state and partly by the lack of certainty of the diagnosis when angiography failed to reveal a malignant circulation in the focal lesion. At operation the brain needle encountered a soft area in the three solid lesions with a gritty feel, presumably due to gliosis and spotty calcification. Resection was carried out with the pathological diagnosis still uncertain, though a colour change (haemosiderin) did sometimes suggest a lesion that had bled. No haematomas were encountered at surgery however. The neuropathological examination of these lesions is also difficult since recent haemorrhage may have destroyed much of the AVM and the gliosis in neighbouring white matter may initially suggest a low grade glioma. The presence of abnormal blood vessels often closely applied to each other with abnormalities of their wall structure is the diagnostic feature. ${ }^{7}$ The lack of angiographic filling is presumably related to the presence of thrombosed vessels or to destructive effects of previous haemorrhage.

Small AVMs are well documented as a cause of cerebral haemorrhage but their presentation with focal epilepsy and a CT appearance suggestive of a low grade glioma needs to be recognised. Surgical excision improved epilepsy control in our cases and is generally recommended. There is a risk that radiotherapy might be preferred if a glioma is diagnosed. Clearly angiographically avascular masses warrant biopsy or excision, and in a young adult with focal fits an occult AVM is a real though rare possibility

We are grateful to Dr HC Grant and Dr AMB Malbouisson for reviewing the histopathological material, and to Dr BE Kendall and Dr I Moseley for the neuroradiological studies. 


\section{References}

1 Olivercona H, Rives J. Arteriovenous aneurysms of the brain. Their diagnosis and treatment. Arch Neurol Psychiatr 1948;59:567-602.

2 Wilkins RH. Natural history of intracranial vascular malformations. Neurosurgery 1985;16:421-30.

3 Krayenbuhl H, Siebenmann R. Small vascular malformations as a cause of primary intracerebral haemorrhage. J Neurosurg 1965;22:7-20.

4 Hashim ASM, Asakura T, Kiochi U, et al. Angiographically occult arteriovenous malformations. Surg
Neurol 1985;16:431-9.

5 Golden JB, Kramer RA. The angiographically occult cerebro-vascular malformation. Report of three cases. J Neurosurg 1978;48:292-6.

6 Kendall BE, Claveria LE. The use of computed axial tomography (CAT) for the diagnosis and management of intracranial angiomas. Neuroradiology 1976;12: 141-60.

7 Wharen PE Jr, Scheithauer BW, Laws ER Jr. Thrombosed arteriovenous malformations of the brain. J Neurosurg 1982;57:520-26. 